QVIPUIKAMAYOC $\mid$ Revista de la Facultad de Ciencias Contables

Vol. 20 N. ${ }^{\circ} 37$ pp. 7-14 (2012) UNMSM, Lima, Perú

ISSN: 1560-9103 (versión impresa) / ISSN: 1609-8196 (versión electrónica)

\title{
EL DILEMA DE LOS COSTOS EN LAS EMPRESAS DE SERVICIOS
}

THE DILEMMA OF COSTS ON BUSINESS SERVICES

Elsa Esther Choy Zevallos *

Docente Asociada de la Facultad de Ciencias Contables - UNMSM

[Recepción: Julio de 2012/ Conformidad: Agosto 2012]

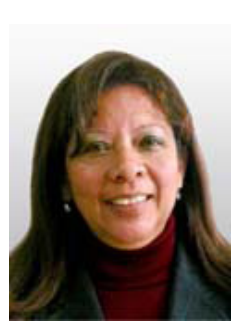

\section{RESUMEN}

En las últimas décadas el sector servicio ha mantenido un crecimiento sostenido en la economía de nuestro país, de manera que un porcentaje significativo de la mano de obra se encuentra empleada en estas organizaciones que presentan características particulares . Sin embargo, cuando nos referimos al tema de costos, ésta se encuentra estrechamente vinculada al sector industrial y permite desarrollar abundante información, mientras que por el sector servicios es escasa la información. El presente trabajo presenta justificación, porque resulta importante establecer un marco teórico que permita conceptualizar las empresas de servicios y sus características y luego enfocarnos en los costos y modelos para el sector servicios.

Palabras Clave: Empresa de servicios, costo de servicio, modelos del costo

\begin{abstract}
In recent decades, the service sector has maintained a steady growth in the economy of our country, so that a significant percentage of the workforce is employed in these companies that have specific characteristics.

However, when it comes to the information costs, it is closely linked to the manufacturing industry and there is a wealth of information, while the service sector provides little information.

It is important to establish a theoretical framework that allows us to conceptualize service companies and its characteristics, and then focus on the costs and models for the services sector.
\end{abstract}

Keywords: Enterprise Services, Service Costs, Cost Models

\footnotetext{
* Doctora en Ciencias Contables y Empresariales y Magister en Dirección Financiera. Contadora Publica Colegiada Certificada. Docente Investigadora de la UNMSM.

E-mail: eschoyz@yahoo.com
} 


\section{INTRODUCCIÓN}

Este estudio, tiene como propósito fundamental proporcionar información para la aplicación y determinación de los costos en empresas que prestan servicios.

Actualmente nos encontramos en una economía globalizada donde las empresas crecen rápidamente y sus costos son más altos y complejos de determinar, especialmente en este sector. Establecer los costos del servicio es uno de los principales desafíos para muchas organizaciones que realizan esta actividad.

La investigación se justifica porque proporciona un marco teórico relacionado con las empresas de servicios, características y peculiaridades, así como señala algunos modelos de costeo para las distintas organizaciones de servicios.

El trabajo plantea en primer término aspectos teóricos como actividades, características, categorías, niveles, tipos, clases, entre otros temas, que se relacionan con las empresas de servicios. Luego, enfoca el tema de los costos de servicios y modelos de costo, y por último presenta las conclusiones y recomendaciones del trabajo.

\section{ASPECTOS GENERALES}

Es preciso señalar algunos conceptos relacionados con el tema y que ayudarán a ubicarnos desde la perspectiva de la teoría de costos

\section{Servicio}

Según Kotler Phillip: "Un servicio es todo acto o función que una parte puede ofrecer a otra, que es esencialmente intangible y no da como resultado ninguna propiedad. Su producción puede o no (...) vincularse a un producto físico."

\section{Costo}

Para Paul Samuelson; "Costo es la suma de erogaciones en que incurre una persona física o moral para la adquisición de un bien o de un servicio, con la intención de que genere ingresos en el futuro."

\section{EMPRESA DE SERVICIOS}

Charles Hornegren, afirma que: "Una empresa de servicios es aquella que a través de diferentes procesos que implican una serie de actividades es capaz de ofrecer o prestar un servicio específico o bien servicios integrados.”

El término servicio es conceptualizado por diversos expertos:

Stanton, Etzel y Walker, definen los servicios como "actividades identificables e intangibles que son el objeto principal de una transacción ideada para brindar a los clientes satisfacción de deseos o necesidades".

Para Richard L. Sandhusen: "Los servicios son actividades, beneficios o satisfacciones que se ofrecen en renta o a la venta, $y$ que son esencialmente intangibles y no dan como resultado la propiedad de algo".

Según Lamb, Hair y McDaniel: "Un servicio es el resultado de la aplicación de esfuerzos humanos o mecánicos a personas u objetos. Los servicios se refieren a un hecho, un desempeño o un esfuerzo que no es posible poseer físicamente"

Para la American Marketing Association (A.M.A.), los servicios son "productos, tales como un préstamo de banco o la seguridad de un domicilio, que son intangibles o por lo menos substancialmente. Si son totalmente intangibles, se intercambian directamente del productor al usuario, no pueden ser transportados o almacenados, y son casi inmediatamente perecederos. Los productos de servicio son a menudo difíciles de iden- 
tificar, porque vienen a existir en el mismo tiempo que se compran y que se consumen. Abarcan los elementos intangibles que son inseparabilidad; que implican generalmente la participación del cliente en una cierta manera importante; no pueden ser vendidos en el sentido de la transferencia de la propiedad; y no tienen ningún título".

Kotler, Bloom y Hayes, definen un servicio de la siguiente manera: "Un servicio es una obra, una realización o un acto que es esencialmente intangible y no resulta necesariamente en la propiedad de algo. Su creación puede o no estar relacionada con un producto físico".

Teniendo en cuenta las anteriores propuestas, se plantea la siguiente definición de servicios:

"Los servicios son actividades identificables, intangibles y perecederas que son el resultado de esfuerzos humanos o mecánicos que producen un hecho, un desempeño o un esfuerzo que implican generalmente la participación del cliente y que no es posible poseer físicamente, ni transportarlos o almacenarlos, pero que pueden ser ofrecidos en renta o a la venta; por tanto, pueden ser el objeto principal de una transacción ideada para satisfacer las necesidades o deseos de los clientes".

\section{CARACTERÍSTICAS DE LAS EMPRE- SAS DE SERVICIOS}

Poseen ciertas características y particularidades, que en algunos casos dificultan su análisis:

- El recurso humano es el principal insumo que se utiliza para proporcionar el servicio, y su cálculo mayormente se esta- blece por el número de horas y tarifa horaria. La mano de obra directa permite estimar los costos indirectos que se atribuyan o se asigne para alcanzar el costo del servicio.

- El producto que se ofrece es intangible, el insumo principal es la venta de información o la realización de alguna actividad física o administrativa a favor del cliente. El producto final se resume en un reporte, donde el costo del papel es insignificante en comparación al valor agregado que tiene intrínsecamente la información que contiene.

- Por lo general se adecúa a un sistema de costos por órdenes, más que procesos, este último no es común que se utilice en estas empresas. El costeo por órdenes, es usado debido a que cada servicio que se pide tiende a ser diferente, por las especificaciones que establece el cliente.

- La orden de un cliente es un trabajo distinto e implica un conjunto de actividades diferentes, con cuenta y número de orden específico. En tal sentido, cada orden o servicio se debe identificar los ingresos y sus respectivos costos directos e indirecto

\section{CATEGORÍAS DE LAS EMPRESAS DE SERVICIOS}

En este tipo de empresas pueden identificarse dos categorías:

- Empresas orientadas a prestar un servicio específico.

- Empresas orientadas a prestar servicios integrados.

Las empresas que prestan servicio específico por lo general están orientadas a ofrecer un servicio a los consumidores de una manera permanente, como los despachos de 
contabilidad, de diseño de ingeniería, de asesoramiento legal, entre otros.

Las empresas orientadas a prestar servicios integrados se caracterizan por ofrecer servicios complementarios entre sí, para satisfacer necesidades más complejas de los clientes, como las compañías de seguros de vida, bancos comerciales, hospitales, empresas de transportes, entre otros.

Aquellas empresas que poseen un alto volumen de servicios ofrecidos, por lo general aquellas que ofrecen servicios integrados, pueden utilizar un sistema de costos por proceso.

\section{CLASIFICACIÓN DE EMPRESAS DE SERVICIOS}

La clasificación de las empresas de servicios obedecen a distintos enfoques:

-Empresas intensivas en capital intelectual: El recurso primordial para la realización del servicio es el conocimiento en las áreas de: especialidad de la empresa. Ejemplo: servicio de asesoría y consultoría contable-tributaria -Empresa intensiva en capital humano: En este tipo de organización se requiere una fuerte inversión de hora-hombre para que el servicio se realice. Ejemplo: Restaurante de comida rápida.

-Empresa intensiva en capital financiero: En este tipo de organización el servicio es provisto gracias a una inversión fuerte en capital. Ejemplo: servicios bancarios.

\section{TIPOS DE EMPRESAS DE SERVICIOS}

-Empresa de servicio de transportes: Proporciona el servicio de traslado o desplazamiento de personas o de cargas.
-Empresa de servicios públicos: Brinda atención a las necesidades de la comunidad.

-Empresa de servicio de seguros: Otorga el servicio de seguridad ante cualquier riesgo. -Empresa de servicio de fianzas: Sirve de intermediario en las garantías.

-Empresa de servicio de créditos: Ofrece soluciones financieras.

\section{ÁREAS EN LA ORGANIZACIÓN DE LAS EMPRESAS DE SERVICIOS}

Por lo general tres áreas:

-El área de operación, en muchas empresas como bancos, hoteles y hospitales es usar la mano de obra, materiales y recursos de la tecnología para satisfacer las necesidades de los clientes.

-Elárea de soporte varía con el tipo de empresa dependiendo el servicio que se ofrece. Por ejemplo, en una línea aérea, el departamento de mantenimiento es el área de soporte, en un hospital es el departamento de archivos y en un banco es el departamento de sistemas de información.

-El área de mercadotecnia y ventas son relevantes en este tipo de empresas, debido a que a través de ellos se contacta al cliente, se promueve el servicio y se desarrollan nuevos servicios.

\section{CARACTERÍSTICAS DE LOS SERVI- CIOS}

Los servicios que tienen como finalidad responder y satisfacer las necesidades del cliente, tiene las siguientes particularidades: -Intangibilidad: Los servicios son intangibles a diferencia de los productos físicos, los servicios no se pueden ver, degustar, tocar, escuchar u oler antes de comprarse. 
-Inseparabilidad: Los servicios por lo regular se producen y consumen simultáneamente. Esto no sucede con los bienes físicos, que se fabrican, se colocan en inventarios, se distribuyen a través de varios intermediarios y se consumen posteriormente.

-Variabilidad: Puesto que los servicios dependen de quien los presta y de donde se prestan, son muy variables. Por ejemplo, algunos doctores saben tratar muy bien a sus pacientes; otros no les tienen tanta paciencia. -Imperdurabilidad: Los servicios no pueden almacenarse. El hecho de que los servicios sean imperdurables no es un problema cuando la demanda es constante, si la demanda fluctúa, las empresas de servicios tienen problemas

\section{CATEGORÍAS DE LOS SERVICIOS}

Los servicios brindados por diversas empresas se clasifican en determinadas categorías, las cuales son:

-El bien tangible puro: La oferta consiste primordialmente en un bien tangible, ejemplo de ello puede ser el jabón o la sal. El producto no va acompañado de servicios.

-El bien tangible con servicios anexos: La oferta consiste en un bien tangible acompañado por uno o más servicios.

-El híbrido: La oferta consiste en bienes y servicios por partes iguales. Por ejemplo, la persona que acude a los restaurantes va tanto por los alimentos como por el servicio.

-Servicio principal con bienes y servicios secundarios anexos: La oferta consiste en un servicio principal acompañado de servicios adicionales o bienes de apoyo.

-El servicio puro: La oferta consiste primordialmente en un servicio. Por ejemplo, podemos citar el cuidado de niños, la psicoterapia y los masajes.

\section{LOS COSTOS EN LAS EMPRESAS DE SERVICIOS}

El reconocimiento de los costos derivados de la prestación de servicios de actividades ordinarias requiere cierta técnica y procedimiento, para lo cual se debe tomar en cuenta todos los desembolsos como: personal, insumos y otros recursos utilizados para brindar dicho servicio, que se caracteriza por ser intangible y de consumo inmediato.

La existencia de un consumo inmediato en las empresas de servicios, por lo general, implica que el cliente participe en el proceso de transformación, generándose de esta forma un elevado uso de mano de obra directa por parte de la empresa prestadora del servicio.

\section{LA NIC 2 para empresas de servicios}

El párrafo 19 de la NIC 2 "Inventarios" establece que en el caso que un prestador de servicios tenga inventarios, los medirá por los costos que suponga su producción. Estos costos se componen fundamentalmente de mano de obra y otros costos del personal directamente involucrado en la prestación del servicio, incluyendo personal de supervisión y otros costos indirectos atribuibles.

La mano de obra y los demás costos relacionados con las ventas, y con el personal de administración general, no se incluirán en el costo de los inventarios, sino que se contabilizarán como gastos del periodo en el que se hayan incurrido.

Los costos de los inventarios de un prestador de servicios no incluirán márgenes de ganancia ni costos indirectos no atribuibles que, a menudo, se tienen en cuenta en los precios facturados por el prestador de servicios. 


\section{SISTEMAS DE COSTOS}

Las empresas de servicios utilizan los sistemas tradicionales: por órdenes de trabajo y por procesos. Gran parte de estas empresas aplican sistemas más modernos como Costos Basados en las Actividades (CBA)

El sistema de costos por órdenes de trabajo consiste en la generación de bienes o servicios heterogéneos, efectuados a la medida del cliente; en ese sentido, los costos se acumulan por cada pedido u orden de los clientes, existiendo una identificación de cada orden de trabajo.

Dentro de las actividades que aplican este sistema se ubican las sociedades de auditoría, los trabajos de publicidad, las imprentas, las actividades de ingeniería y diseño, reparación de máquinas, entre otras.

El sistema de costos por procesos permite que se acumulen los costos de producción por departamento o centro de costos, y es aplicable para los prestadores de servicios homogéneos, de naturaleza continua e ininterrumpida, en donde es difícil diferenciar una unidad de servicio de las demás.

Dentro de las actividades que pueden aplicar este tipo de costos se encuentran las empresas de servicios públicos, las sociedades agentes de bolsa, las empresas del sistema financiero, salas de cine, entre otras.

\section{ELEMENTOS DEL COSTO DE SERVI- CIOS}

-Costo directos: Los que pueden identificarse específicamente en la unidad.

-Costos indirectos: No puede identificarse en la unidad.

-Costos operacionales: Los costos en que incurre un sistema ya instalado o adquirido durante su vida útil, con objeto de realizar los procesos de producción, se denominan costos de operación, e incluyen los necesarios para el manteni- miento del sistema. Dentro de los costos de operación más importantes tenemos los siguientes:

a) Gastos técnicos y administrativos. Son aquellos que representan la estructura ejecutiva, técnica y administrativa de una empresa, tales como, jefes de compras, almacenistas, mecánicos, veladores, dibujantes, ayudantes, mozos de limpieza y envíos, etc.

b) Alquileres y/o depreciaciones. Son aquellos gastos por conceptos de bienes muebles e inmuebles, así como servicios necesarios para el buen desempeño de las funciones ejecutivas, técnicas y administrativas de una empresa, tales como: rentas de oficinas y almacenes, servicios de teléfonos, etc.

c) Obligaciones y seguros. Son aquellos gastos obligatorios para la operación de la empresa y convenientes para la dilución de riesgos a través de seguros que impidan una súbita descapitalización por siniestros. Entre estos podemos enumerar: inscripción a la Cámara Nacional de la Industria de la Construcción, registro ante la Secretaría del Patrimonio Nacional, Seguros de Vida, etc.

d) Materiales de consumo. Son aquellos gastos en artículos de consumo, necesarios para el funcionamiento de la empresa, tales como: combustibles y lubricantes de automóviles y camionetas al servicio de las oficinas de la planta, gastos de papelería impresa, artículos de oficina, etc.

e) Capacitación y Promoción. Todo colaborador tiene el derecho de capacitarse y pensamos que en tanto éste lo haga, en esa misma medida, o mayor aún, la empresa mejorará su productividad. Entre los gastos de capacitación y promoción podemos mencionar: cursos a obreros y empleados, gastos de actividades deportivas, de celebraciones de oficinas, etc. 


\section{DETERMINACIÓN DE LOS COSTOS EN EMPRESAS DE SERVICIOS}

La determinación de los costos en las empresas de servicios es más compleja y diversa, ya que los procedimientos de cálculo depende del tipo de actividad, y son diversos los servicios. Por ejemplo, el proceso de determinar el costo en una empresa de seguros es diferente de una empresa de transporte, banco o servicio profesional.

Es decir, para determinar los costos de servicios, es necesario tomar en consideración las características propias de cada empresa, aunque en términos generales, las empresas de servicios se caracterizan por lo siguiente: -Los servicios producidos no pueden ser inventariados para venderlos en el futuro, de manera que los sistemas de contabilidad de gestión de la mayoría de las organizaciones de servicios no tienen que preocuparse de elaborar periódicamente informes de valoración de inventarios, como lo hacen las empresas manufactureras.

-Las normas tradicionales de elaboración de informes financieros limitan, de alguna manera, la estructura de los informes de costos en las organizaciones de servicios, por lo que éstas deben establecer sus propios modelos de informe.

-La producción es difícil de medir, ya que las unidades de medidas de los servicios son menos obvias que las empresas de fabricación, debiendo valerse de unidades mixtas, tales como metros cúbicos, kilómetros cuadrados, días.

-Los costos indirectos de fabricación de las empresas que fabrican productos equivalen a los costos indirectos de producir servicios, en las empresas de servicios

\section{MODELOS DE COSTOS PARA EMPRE- SAS DE SERVICIOS}

Los modelos de costos que pueden ser utilizados en las empresas de servicios no difieren de las que utilizan las empresas industriales o comerciales, sin embargo, cualquiera de los modelos planteados, ya sea en su forma pura o combinada pueden ser aplicados en las mismas condiciones. Aún cuando muchos especialistas recomiendan que para las empresas de servicios resulta más oportuno trabajar con un modelo de acumulación por órdenes, es posible que muchos servicios que se han automatizado y seriado se adecúen a la aplicación de un modelo de costos por procesos.

Asimismo, una de las características de los servicios es la heterogeneidad, lo que complica la aplicación de un modelo normalizado. La automatización ha favorecido también la normalización de los procesos, motivo por el cual es posible tender a una estandarización de los costos.

\section{CONCLUSIONES}

1. Las empresas de que prestan servicios tienen características particulares que condicionan su gestión.

2. La determinación de los costos en las empresas de servicios resulta compleja y diversa, debido a que los procedimientos para su cálculo depende del tipo de actividad, las mismas que resultas muy variadas como: transporte, salud, educación, gastronomía, servicios profesionales, entre otras. 


\section{RECOMENDACIONES}

1.Es importante realizar un estudio y análisis macroeconómico del sector servicios, ya que permite conocer ciertos comportamientos que ayudan a comprender su funcionamiento, características y particularidades.

2.Para el cálculo de los costos de servicios, es necesario realizar una interpretación previa de las condiciones del proceso técnicoorganizativo y a partir del mismo definir los modelos de costos que brindarán sustento a las técnicas de costeo específicas que se apliquen.

\section{REFERENCIAS BIBLIOGRÁFICAS}

1. Aguirre, J ( 2004). Sistema de costeo. La asignación del costo total a productos y ser- vicios. Universidad de Bogotá Jorge Tadeo Lozano. Colombia

2. Cuervo, J (2006). Costeo basados en Actividades $A B C$ (Gestión basada en actividades $A B M)$. Ecoe Ediciones. Perú.

3. Cuevas, C. Contabilidad de Costos (Enfoque gerencial y de gestión). Quórum ediciones. Colombia. Disponible en: http://www.intercostos.org/documentos/300.pdf

4. Hansen, R.(.2007). Administración de costos, Contabilidad y control. Cengage Editores. México. Disponible en: http:// www.monografias.com/trabajos31/costeo-abc/costeo-abc.shtml 